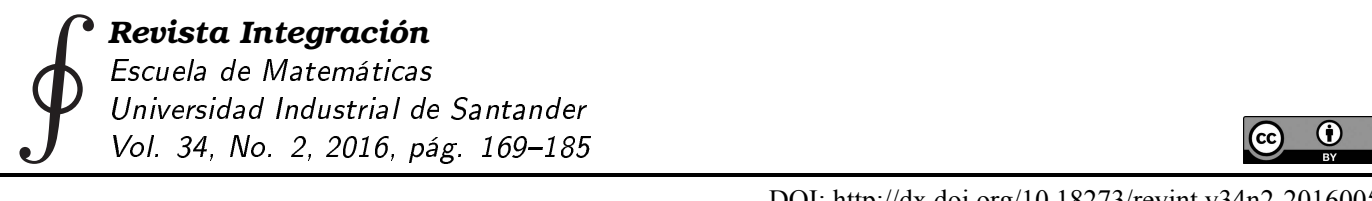

DOI: http://dx.doi.org/10.18273/revint.v34n2-2016005

\title{
Un modelo de redes neuronales para complementariedad no lineal
}

\author{
Favián Arenas*, Rosana Pérez, Hevert Vivas \\ Universidad del Cauca, Departamento de Matemáticas, Popayán, Colombia.
}

Resumen. En este artículo presentamos un modelo de red neuronal para resolver el problema de complementariedad no lineal. Para ello, reformulamos este problema como uno de minimización sin restricciones usando una familia uniparamétrica de funciones de complementariedad. Demostramos resultados de existencia y convergencia de la trayectoria de la red neuronal, así como resultados de estabilidad en el sentido de Lyapunov, estabilidad asintótica y exponencial. Además, presentamos resultados numéricos preliminares que ilustran un buen desempeño práctico del modelo.

Palabras clave: Red neuronal, problema de complementariedad no lineal, estabilidad, reformulación.

MSC2010: 90C30, 90C33, 90C53, 90B10.

\section{A neural network model for nonlinear complementarity problems}

\begin{abstract}
In this paper we present a neural network model for solving the nonlinear complementarity problem. This model is derived from an equivalent unconstrained minimization reformulation of the complementarity problem, which is based on a one-parametric class of nonlinear complementarity functions. We establish the existence and convergence of the trajectory of the neural network, and we study its Lyapunov stability, asymptotic stability as well as exponential stability. Numerical tests verify the obtained theoretical results.

Keywords: Neural network, nonlinear complementarity problem, stability, reformulation.

\footnotetext{
* E-mail: farenas @unicauca.edu.co

Recibido: 22 de junio de 2016, Aceptado: 21 de noviembre de 2016.

Para citar este artículo: F. Arenas, R. Pérez, H. Vivas, Un modelo de redes neuronales para complementariedad no lineal, Rev. Integr. Temas Mat. 34 (2016), No. 2, 169-185.
} 


\section{Introducción}

Dada $F: \mathbb{R}^{n} \rightarrow \mathbb{R}^{n}$ continuamente diferenciable, el Problema de Complementariedad No Lineal (PCNL) consiste en encontrar un vector $\boldsymbol{x} \in \mathbb{R}^{n}$ que satisfaga las tres condiciones siguientes,

$$
\boldsymbol{x} \geq 0, \quad F(\boldsymbol{x}) \geq 0, \quad \boldsymbol{x}^{T} F(\boldsymbol{x})=0,
$$

donde la expresión $\boldsymbol{y} \geq 0$, cuando $\boldsymbol{y} \in \mathbb{R}^{n}$, significa que $y_{i} \geq 0$ para todo $i=1, \ldots, n$.

Este problema surge naturalmente en ingeniería, física y economía, entre otros campos ([12], [16], [24]), por lo cual ha habido gran interés en el estudio y creación de métodos que permitan resolverlo. Entre dichos métodos están, por una parte, los de homotopía derivados de los de punto fijo $([11],[26])$. Por otra parte, están los métodos (quizá, los más populares) de reformulación que plantean el PCNL como un problema de minimización sin restricciones usando una función de mérito $([9],[10],[15])$. Este problema puede resolverse usando diferentes métodos de optimización, como los de tipo Newton $([9],[15])$ o cuasi-Newton ([1], [3]). En los de reformulación se utiliza una función $\varphi: \mathbb{R}^{2} \rightarrow \mathbb{R}$ tal que $\varphi(a, b)=0 \Longleftrightarrow a \geq 0, b \geq 0, a b=0$, conocida como función de complementariedad ([7], [13], [15], [22]).

No obstante, en algunas aplicaciones se necesitan soluciones del PCNL en tiempo real, para lo cual las técnicas estándares de optimización no son muy apropiadas por el alto costo computacional que demandan. En este caso, una técnica útil consiste en usar redes neuronales artificiales ([6],[14], [17]) como, por ejemplo, las llamadas redes de Hopfield para resolver el problema de optimización mencionado en el párrafo anterior. La idea principal de esta técnica consiste en construir una función no negativa, llamada función de energía, y un sistema dinámico que represente la red neuronal artificial, que normalmente se plantea en forma de una ecuación diferencial ordinaria de primer orden con una condición inicial. Se espera que la trayectoria solución del sistema se aproxime, a partir de un estado inicial, a un estado estático (o punto de equilibrio) que corresponde a la solución del problema de optimización subyacente ([14]).

En particular, en [6] y [17] se presentan dos propuestas que incorporan la técnica de solución al PCNL mediante redes neuronales artificiales. Para ello, se usan, por un lado, funciones de complementariedad para construir la función a minimizar, que es la función energía del modelo, y, por otro lado el método de máximo descenso ([10], [19]) para modelar la red neuronal (una método natural en un problema de minimización). En [17] se usa la función de complementariedad de Fischer-Burmeinster ([15]) definida por

$$
\varphi(a, b)=\|(a, b)\|_{2}-a-b,
$$

y, en [6], se usa la función de complementariedad de Fischer-Burmeinster generalizada ([7]) definida por

$$
\varphi_{p}(a, b)=\|(a, b)\|_{p}-a-b, \quad p>1 .
$$

En ambos trabajos se analiza teórica y numéricamente el modelo propuesto.

Motivados por los resultados de los trabajos anteriores, y en especial por las propiedades de la familia uniparamétrica de funciones de complementariedad ([2],[15]) definida por

$$
\varphi_{\lambda}(a, b)=\sqrt{(a-b)^{2}+\lambda a b}-a-b, \quad \lambda \in(0,4),
$$

[Revista Integración 
proponemos un modelo de red neuronal de Hopfield que usa, por primera vez, la familia uniparamétrica (1) para construir la función de energía del modelo. Tal como lo hacen los autores de [6] y [17], usamos el método de máximo descenso para modelar la red neuronal. Cabe mencionar que nuestro modelo es más general que el propuesto en [17], ya que la función que allí se usa es el miembro de la familia (1) correspondiente a $\lambda=2$. Además, cuando $\lambda$ tiende a cero, $\varphi_{\lambda}(a, b)$ tiende a un múltiplo de una de las funciones de complementariedad más utilizadas en el contexto de problemas de complementariedad no lineal mediante reformulación, la llamada función mínimo ([22]), definida por $\varphi(a, b)=\operatorname{mín}\{a, b\}$. Algoritmos que usan esta función han reportado buenas propiedades de convergencia local ([20], [21], [22]), mientras que aquellos que usan la función de Fischer-Burmeinster tienen buenas propiedades de convergencia global ([3], [15]). Aprovechamos estas ventajas incorporarando un parámetro $\lambda$ dinámico ([15]) en el algoritmo del modelo que proponemos, lo cual resulta más eficiente que usar un parámetro fijo, como se evidencia en los resultados numéricos que presentamos al final del artículo, donde comparamos la simulación numérica de nuestro modelo con la del modelo propuesto en [17].

Las funciones de complementariedad mencionadas son funciones no diferenciables. Por ello, para el análisis de estabilidad y convergencia de la trayectoria un modelo que use dichas funciones debe acudirse a teoría de funciones no diferenciables. En particular, la reformulación del PCNL basada en la familia (1) posee propiedades ([1], [15]) análogas a las de las reformulaciones utilizadas en [6] y [17], lo que fue de gran utilidad en el análisis de estabilidad y convergencia del modelo que proponemos en el presente artículo.

Organizamos la presentación de este artículo en la siguiente forma. En la Sección 2, reformulamos el problema de complementariedad como un problema de minimización usando la familia de funciones de complementariedad propuesta en [15], y proponemos un modelo de red neuronal artificial para resolverlo. En la Sección 3 presentamos, de manera preliminar, algunos conceptos necesarios para el desarrollo teórico posterior. En la Sección 4 realizamos el análisis de estabilidad y convergencia del modelo. En la Sección 5 analizamos el comportamiento numérico del modelo propuesto. Finalmente, en la Sección 6 hacemos algunos comentarios finales y propuestas de trabajos futuros sobre el tema.

\section{Reformulación del PCNL como un problema de minimización}

Usando la familia de funciones de complementariedad (1) podemos definir la función $\Phi_{\lambda}: \mathbb{R}^{n} \rightarrow \mathbb{R}^{n}$ y reformular el PCNL como un sistema de ecuaciones no lineales, el cual resulta no diferenciable debido a la no diferenciabilidad de $\varphi_{\lambda}$ :

$$
\Phi_{\lambda}(\boldsymbol{x})=\left(\begin{array}{c}
\varphi_{\lambda}\left(x_{1}, F_{1}(\boldsymbol{x})\right) \\
\vdots \\
\varphi_{\lambda}\left(x_{n}, F_{n}(\boldsymbol{x})\right)
\end{array}\right)=0 .
$$

A partir de la definición de función de complementaridad tenemos que un vector $\boldsymbol{x}_{*}$ es solución del PCNL si, y solo si, $\boldsymbol{x}_{*}$ es solución del sistema de ecuaciones no lineales no diferenciable $(2)$.

Si $\Phi_{\lambda}$ es continua según Lipschitz su jacobiano generalizado existe ([8], [15]) y está defi-

Vol. 34, No. 2, 2016] 
nido en un punto $\boldsymbol{x} \in \mathbb{R}^{n}$ como el conjunto,

$$
\partial \Phi_{\lambda}(\boldsymbol{x})=\operatorname{conv}\left\{\lim _{k \rightarrow \infty} \Phi_{\lambda}^{\prime}\left(\boldsymbol{x}_{k}\right) \in \mathbb{R}^{n \times n}: \boldsymbol{x}_{k} \rightarrow \boldsymbol{x}, \boldsymbol{x}_{k} \in D_{\Phi_{\lambda}}\right\},
$$

donde $D_{\Phi_{\lambda}}$ es el conjunto de todos los puntos de $\mathbb{R}^{n}$ en los que la función $\Phi_{\lambda}$ es diferenciable y $\operatorname{conv}\{A\}$ representa la envolvente convexa del conjunto $A$.

Por otra parte, si definimos la función de mérito ([10]) $E_{\lambda}: \mathbb{R}^{n} \rightarrow \mathbb{R}^{n}$ como

$$
E_{\lambda}(\boldsymbol{x})=\frac{1}{2}\left\|\Phi_{\lambda}(\boldsymbol{x})\right\|^{2},
$$

tenemos que (2) es equivalente a $E_{\lambda}\left(\boldsymbol{x}_{*}\right)=0$, lo que a su vez es equivalente a que $\boldsymbol{x}_{*}$ es solución del PCNL. Por lo tanto, resolver el PCNL es equivalente a encontrar un minimizador global del problema de minimización sin restricciones:

$$
\begin{gathered}
\text { Minimizar } E_{\lambda}(\boldsymbol{x}), \\
\boldsymbol{x} \in \mathbb{R}^{n},
\end{gathered}
$$

el cual es un problema de optimización diferenciable. En efecto, en [15] se demuestra que la función $E_{\lambda}$ es continuamente diferenciable y que, para cualquier $V \in \partial \Phi_{\lambda}(\boldsymbol{x})$,

$$
\nabla E_{\lambda}(\boldsymbol{x})=V^{T} \Phi_{\lambda}(\boldsymbol{x}) .
$$

Para resolver el problema de minimización (5) y, dado que $E_{\lambda}$ es continuamente diferenciable, proponemos un modelo de red neuronal de la forma

$$
\frac{d \boldsymbol{x}(t)}{d t}=-\rho \nabla E_{\lambda}(\boldsymbol{x}(t)), \quad \boldsymbol{x}(0)=\boldsymbol{x}_{0},
$$

donde $\rho>0$ es un factor de escala que por simplicidad en el análisis teórico del modelo lo tomaremos como 1. Este modelo es análogo al usado en [6] y [17], porque usa el método de máximo descenso para modelar la red neuronal; pero se diferencia en que su función de energía es la familia de funciones de complementariedad (1), lo que lo convierte en un modelo más general que el propuesto en [17].

\section{Preliminares}

En esta sección incluimos algunos conceptos que serán de utilidad para el desarrollo teórico posterior. Iniciamos con dos definiciones relacionadas con los conceptos de matriz y función $P_{0}$ ([6], [15], [17]).

Definición 3.1. Se dice que una matriz $M \in \mathbb{R}^{n \times n}$ es una matriz $P_{0}$ si todos sus menores principales son no negativos.

Definición 3.2. Una función $F: \mathbb{R}^{n} \rightarrow \mathbb{R}^{n}$ es una función $P_{0}$, si para todo $\boldsymbol{x}, \boldsymbol{y} \in \mathbb{R}^{n}$ con $\boldsymbol{x} \neq \boldsymbol{y}$, se satisface que máx $\left(x_{i}-y_{i}\right)\left[F_{i}(\boldsymbol{x})-F_{i}(\boldsymbol{y})\right] \geq 0, i=1, \ldots, n$.

En lo que sigue, presentamos conceptos relacionados con estabilidad de ecuaciones diferenciales ordinarias de primer orden (ver [27]).

Consideremos el siguiente sistema dinámico ([27]), donde $f: \mathbb{R}^{n} \rightarrow \mathbb{R}^{n}$ es una función continua

$$
\frac{d \boldsymbol{x}(t)}{d t}=f(\boldsymbol{x}(t)), \quad \boldsymbol{x}\left(t_{0}\right)=\boldsymbol{x}_{0} .
$$


Definición 3.3. Un vector $\boldsymbol{x}_{*}=\boldsymbol{x}\left(t_{*}\right) \in \mathbb{R}^{n}$ es llamado un punto de equilibrio o un estado estacionario de (8), si $f\left(\boldsymbol{x}_{*}\right)=0$. Si existe una vencindad $\Omega^{*} \subseteq \mathbb{R}^{n}$ de $\boldsymbol{x}_{*}$ tal que $f\left(\boldsymbol{x}_{*}\right)=0$ y $f(\boldsymbol{x}) \neq 0$ para todo $\boldsymbol{x} \in \Omega^{*} \backslash\left\{\boldsymbol{x}_{*}\right\}$, entonces $\boldsymbol{x}_{*}$ es llamado un punto de equilibrio aislado.

Si en (8), $f=\nabla E_{\lambda}$, obtenemos el modelo de red neuronal (7) propuesto. Así, de acuerdo con la Definición 3.3 , un punto $\boldsymbol{x} \in \mathbb{R}^{n}$ es un punto de equilibrio de (7) si $\nabla E_{\lambda}(\boldsymbol{x})=0$. Es decir, un punto de equilibrio de (7) es un punto estacionario de $\nabla E_{\lambda}$.

Teorema 3.4 (Existencia y unicidad). Para cualesquiera $t_{0} \geq 0$ y $\boldsymbol{x}_{0} \in \mathbb{R}^{n}$ existe una solución local $\boldsymbol{x}(t), t \in\left[t_{0}, \tau\right)$ de (8) para algún $\tau>t_{0}$. Además, si $f$ es localmente continua según Lipschitz en $\boldsymbol{x}_{0}$, entonces la solución es única; si $f$ es continua según Lipschitz en $\mathbb{R}^{n}$, entonces $\tau$ puede extenderse $a+\infty$.

Si una solución local definida en $\left[t_{0}, \tau\right)$ no se puede extender a una solución local en un intervalo más grande $\left[t_{0}, \tau_{1}\right)$, con $\tau_{1}>\tau$, entonces es llamada una solución maximal, y el intervalo $\left[t_{0}, \tau\right)$ es el intervalo maximal de existencia. Una solución local arbitraria tiene una extensión a una maximal. El intervalo maximal de existencia asociado con $\boldsymbol{x}_{0}$ frecuentemente se denota por $\left[t_{0}, \tau\left(x_{0}\right)\right)$.

Teorema 3.5. Si $\boldsymbol{x}(t)$ con $t \in\left[t_{0}, \tau\left(\boldsymbol{x}_{0}\right)\right)$ es una solución maximal y $\tau\left(\boldsymbol{x}_{0}\right)<+\infty$, entonces

$$
\lim _{t \uparrow \tau\left(x_{0}\right)}\|\boldsymbol{x}(t)\|=+\infty
$$

En otras palabras, si $\tau\left(x_{0}\right)<+\infty$, la curva $\boldsymbol{x}(t)$ es inestable, como lo ilustra la Figura 1 .

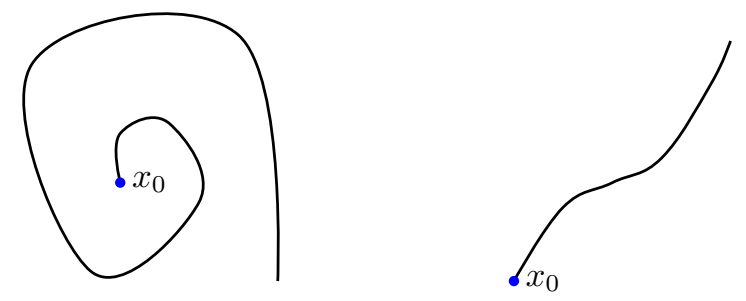

Figura 1. Situaciones que pueden ocurrir cuando $\operatorname{lím}_{t \uparrow \tau(\boldsymbol{x})}\|\boldsymbol{x}(t)\|=+\infty$.

Definición 3.6 (Estabilidad en el sentido de Lyapunov). Sea $\boldsymbol{x}(t)$ una solución de (8). Un punto de equilibrio aislado $\boldsymbol{x}_{*}$ es estable según Lyapunov si para cualquier $\boldsymbol{x}_{0}=$ $\boldsymbol{x}\left(t_{0}\right)$ y cualquier escalar $\epsilon>0$, existe $\delta>0$ tal que si $\left\|\boldsymbol{x}\left(t_{0}\right)-\boldsymbol{x}_{*}\right\|<\delta$, entonces $\left\|\boldsymbol{x}(t)-\boldsymbol{x}_{*}\right\|<\epsilon$, para $t \geq t_{0}$.

Definición 3.7 (Estabilidad asintótica). Un punto de equilibrio aislado $\boldsymbol{x}_{*}$ es estable asintóticamente si, además de ser estable según Lyapunov, cumple que $\boldsymbol{x}(t) \rightarrow \boldsymbol{x}_{*}$ cuando $t \rightarrow \infty$ si $\left\|\boldsymbol{x}\left(t_{0}\right)-\boldsymbol{x}_{*}\right\|<\delta$.

Definición 3.8 (Función de Lyapunov). Sea $\Omega \subseteq \mathbb{R}^{n}$ una vecindad abierta de $\overline{\boldsymbol{x}}$. Una función continuamente diferenciable $\xi: \mathbb{R}^{n} \longrightarrow \mathbb{R}$ es una función de Lyapunov en el

Vol. 34, No. 2, 2016] 
estado $\overline{\boldsymbol{x}}$ (sobre el conjunto $\Omega$ ) para (8), si satisface las dos condiciones siguientes:

$$
\begin{array}{r}
\xi(\overline{\boldsymbol{x}})=0, \xi(\boldsymbol{x})>0, \quad \text { para todo } \boldsymbol{x} \in \Omega, \boldsymbol{x} \neq \overline{\boldsymbol{x}} \\
\frac{d \xi(\boldsymbol{x}(t))}{d t}=\left[\nabla_{\boldsymbol{x}(t)} \xi(\boldsymbol{x}(t))\right]^{T} f(\boldsymbol{x}(t)) \leq 0, \quad \text { para todo } \boldsymbol{x} \in \Omega .
\end{array}
$$

Una función de Lyapunov es llamada frecuentemente una función de energía para (8). El siguiente resultado relaciona estabilidad y función de Lyapunov.

Teorema 3.9. (i) Un punto de equilibrio aislado $\boldsymbol{x}_{*}$ es estable según Lyapunov si existe una función de Lyapunov sobre alguna vecindad abierta de $\boldsymbol{x}_{*}$.

(ii) Un punto de equilibrio aislado $\boldsymbol{x}_{*}$ es estable asintóticamente si existe una función de Lyapunov sobre alguna vecindad $\Omega$ de $\boldsymbol{x}_{*}$ que satisface

$$
\frac{d \xi(\boldsymbol{x}(t))}{d t}<0 \quad \text { para todo } \quad \boldsymbol{x}(t) \in \Omega, \boldsymbol{x}(t) \neq \boldsymbol{x}_{*} .
$$

Una noción más fuerte que la estabilidad según Lyapunov es la llamada estabilidad exponencial.

Definición 3.10 (Estabilidad exponencial). Un punto de equilibrio aislado $\boldsymbol{x}_{*}$ es estable exponencialmente para (8) si existen constantes $\omega<0, \kappa>0$ y $\delta>0$ tales que una solución arbitraria $\boldsymbol{x}(t)$ de (8) con $\left\|\boldsymbol{x}\left(t_{0}\right)-\boldsymbol{x}_{*}\right\|<\delta$ está definida en $[0, \infty)$ y satisface $\left\|\boldsymbol{x}(t)-\boldsymbol{x}_{*}\right\| \leq \kappa e^{\omega t}\left\|\boldsymbol{x}\left(t_{0}\right)-\boldsymbol{x}_{*}\right\|, t \geq t_{0}$.

Observemos que todo punto exponencialmente estable es, a su vez, asintóticamente estable.

\section{Análisis de estabilidad}

Analizamos a continuación el comportamiento de la trayectoria solución del modelo de red neuronal propuesto (7). Estudiamos su existencia y unicidad. Además, para un punto de equilibrio aislado establecemos tres tipos de estabilidad. En primer lugar, analizamos la relación entre un punto de equilibrio de (7) y una solución del PCNL.

Proposición 4.1. $\quad$ (i) Toda solución del PCNL es un punto de equilibrio de (7).

(ii) Si F es una función $P_{0}$ entonces todo punto de equilibrio de (7) es una solución del problema PCNL asociado a $F$.

Demostración. (i) $\mathrm{Si} \boldsymbol{x}$ es una solución del PCNL, entonces $\Phi_{\lambda}(\boldsymbol{x})=0$, y por (6), $\nabla E_{\lambda}(\boldsymbol{x})=0$. Por lo tanto, $\boldsymbol{x}$ es un punto de equilibrio de (7).

(ii) Si $\boldsymbol{x}$ es un punto de equilibrio de (7), entonces $\nabla E_{\lambda}(\boldsymbol{x})=0$, y por tanto, $\boldsymbol{x}$ es un punto estacionario de $E_{\lambda}$; y dado que $F$ es una función $P_{0}$, se tiene que su matriz jacobiana $F^{\prime}(\boldsymbol{x})$ es una matriz $P_{0}$, lo cual garantiza que el punto estacionario $\boldsymbol{x}$ es una solución de PCNL [15]. 
El siguiente es un lema técnico que usaremos en la prueba de existencia y unicidad de la trayectoria solución de (7).

Lema 4.2. Si F es continua según Lipschitz en $\mathbb{R}^{n}$, entonces $\Phi_{\lambda}$ es continua según Lipschitz en $\mathbb{R}^{n}$.

Demostración. Usaremos las equivalencias entre las normas euclidiana $(\|\cdot\|)$ e infinito $\left(\|\cdot\|_{\infty}\right)([25])$; la definición de norma vectorial infinito y la continuidad según Lipschitz de $\phi_{\lambda}$ (ver [1]). Sean $\boldsymbol{x}$ y $\boldsymbol{y}$ en $\mathbb{R}^{n}$. Para algún $1 \leq k \leq n$,

$$
\begin{aligned}
\left\|\Phi_{\lambda}(\boldsymbol{x})-\Phi_{\lambda}(\boldsymbol{y})\right\| & \leq \sqrt{n}\left\|\Phi_{\lambda}(\boldsymbol{x})-\Phi_{\lambda}(\boldsymbol{y})\right\|_{\infty}=\sqrt{n}\left|\varphi_{\lambda}\left(x_{k}, F_{k}(\boldsymbol{x})\right)-\varphi_{\lambda}\left(y_{k}, F_{k}(\boldsymbol{y})\right)\right| \\
& \leq \sqrt{n} \mu\left\|\left(x_{k}-y_{k}, F_{k}(\boldsymbol{x})-F_{k}(\boldsymbol{y})\right)\right\|_{\infty} \\
& \leq \sqrt{n} \mu \operatorname{máx}\left\{\left|x_{k}-y_{k}\right|,\left|F_{k}(\boldsymbol{x})-F_{k}(\boldsymbol{y})\right|\right\} .
\end{aligned}
$$

Si máx $\left\{\left|x_{k}-y_{k}\right|,\left|F_{k}(\boldsymbol{x})-F_{k}(\boldsymbol{y})\right|\right\}=\left|x_{k}-y_{k}\right|$,

$\left\|\Phi_{\lambda}(\boldsymbol{x})-\Phi_{\lambda}(\boldsymbol{y})\right\| \leq \sqrt{n} \mu\left|x_{k}-y_{k}\right| \leq \sqrt{n} \mu\|\boldsymbol{x}-\boldsymbol{y}\|_{\infty} \leq \sqrt{n} \mu\|\boldsymbol{x}-\boldsymbol{y}\|=\gamma_{1}\|\boldsymbol{x}-\boldsymbol{y}\|$.

Si máx $\left\{\left|x_{k}-y_{k}\right|,\left|F_{k}(\boldsymbol{x})-F_{k}(\boldsymbol{y})\right|\right\}=\left|F_{k}(\boldsymbol{x})-F_{k}(\boldsymbol{y})\right|$,

$$
\begin{aligned}
\left\|\Phi_{\lambda}(\boldsymbol{x})-\Phi_{\lambda}(\boldsymbol{y})\right\| & \leq \sqrt{n} \mu\left|F_{k}(\boldsymbol{x})-F_{k}(\boldsymbol{y})\right| \leq \sqrt{n} \mu\|F(\boldsymbol{x})-F(\boldsymbol{y})\|_{\infty} \\
& \leq \sqrt{n} \mu \delta\|\boldsymbol{x}-\boldsymbol{y}\|_{\infty} \leq \sqrt{n} \mu \delta\|\boldsymbol{x}-\boldsymbol{y}\|=\gamma_{2}\|\boldsymbol{x}-\boldsymbol{y}\|,
\end{aligned}
$$

donde $\delta$ es la constante de Lipschitz de la función $F$, con lo que se concluye la prueba.

El siguiente resultado garantiza la existencia y unicidad de una trayectoria solución del modelo propuesto.

Teorema 4.3. (i) Para un estado inicial arbitrario $x_{0}$ existe una solución maximal $\boldsymbol{x}(t)$, con $t \in\left[t_{0} ; \tau\left(x_{0}\right)\right)$, de $(7)$.

(ii) Si $F$ es continua según Lipschitz, entonces la solución maximal es única y $\tau\left(\boldsymbol{x}_{0}\right)=$ $+\infty$.

(iii) Si el conjunto de nivel asociado a $\boldsymbol{x}_{0}, \quad \mathcal{L}\left(\boldsymbol{x}_{0}\right)=\left\{\boldsymbol{x} \in \mathbb{R}^{n}: E_{\lambda}(\boldsymbol{x}) \leq E_{\lambda}\left(\boldsymbol{x}_{0}\right)\right\}$ es acotado, entonces $\tau\left(\boldsymbol{x}_{0}\right)=+\infty$.

Demostración. (i) Sea $\boldsymbol{x}_{0} \in \mathbb{R}^{n}$ un estado inicial arbitrario. Dado que $E_{\lambda}$ es continuamente diferenciable, se tiene que $\nabla E_{\lambda}$ es una función continua. Luego, por el Teorema 3.4, existe una solución maximal de (7).

(ii) Supongamos que $F$ es continua según Lipschitz en $\mathbb{R}^{n}$. Entonces por Lema 4.2 tenemos que $\Phi_{\lambda}$ es continua según Lipschitz en $\mathbb{R}^{n}$. Usando este resultado y (6); tenemos que para $\boldsymbol{x}$ y $\boldsymbol{y}$ en $\mathbb{R}^{n}$ y $\|\cdot\|$ una norma inducida sobre $\mathbb{R}^{n}$,

$$
\begin{aligned}
\left\|\nabla E_{\lambda}(\boldsymbol{x})-\nabla E_{\lambda}(\boldsymbol{y})\right\| & =\left\|V^{T} \Phi_{\lambda}(\boldsymbol{x})-V^{T} \Phi_{\lambda}(\boldsymbol{y})\right\| \leq\|V\|\left\|\Phi_{\lambda}(\boldsymbol{x})-\Phi_{\lambda}(\boldsymbol{y})\right\| \\
& \leq \gamma\|V\|\|\boldsymbol{x}-\boldsymbol{y}\|=\eta\|\boldsymbol{x}-\boldsymbol{y}\|,
\end{aligned}
$$

donde $\gamma$ es la constante de Lipschitz para $\Phi_{\lambda}\left(\gamma=\gamma_{1}\right.$ ó $\left.\gamma=\gamma_{2}\right)$, y $\eta=\gamma\|V\|$. Por lo tanto, $E_{\lambda}$ también es continua según Lipschitz en $\mathbb{R}^{n}$. Por Teorema 3.4 concluimos que la solución maximal es única y $\tau\left(\boldsymbol{x}_{0}\right)=+\infty$.

Vol. 34, No. 2, 2016] 
(ii) Supongamos ahora que el conjunto $\mathcal{L}\left(\boldsymbol{x}_{0}\right)$ es acotado y $\tau\left(\boldsymbol{x}_{0}\right)<+\infty$. Por el Teorema 3.5 tenemos que $\operatorname{lím}_{t \uparrow \tau(\boldsymbol{x})}\|\boldsymbol{x}(t)\|=+\infty$, lo cual implica que la trayectoria $\boldsymbol{x}(t)$ no converge a ningún punto de acumulación. Dado que $\mathcal{L}\left(\boldsymbol{x}_{0}\right)$ es acotado, podemos definir $\tau_{0}$ de la siguiente forma (Figura 2):

$$
\tau_{0}=\inf \left\{s \geq 0 \mid s<\tau\left(\boldsymbol{x}_{0}\right), \boldsymbol{x}(s) \in \mathbb{R}^{n}-\mathcal{L}\left(\boldsymbol{x}_{0}\right)\right\}<+\infty
$$

por hipótesis, $\mathcal{L}\left(\boldsymbol{x}_{0}\right)$ es acotado, y por la continuidad de $E_{\lambda}$ es un conjunto

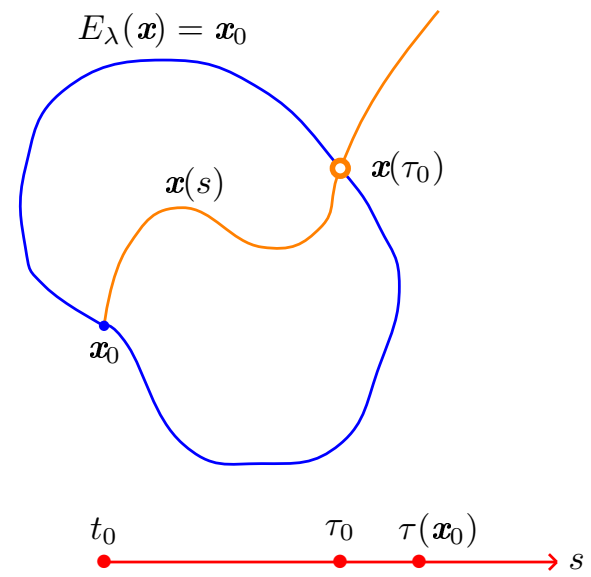

Figura 2. Trayectoria de la solución $\boldsymbol{x}(t)$ cuando se supone que $\tau\left(\boldsymbol{x}_{0}\right)<+\infty$.

cerrado; entonces $\boldsymbol{x}\left(\tau_{0}\right) \in \mathcal{L}\left(\boldsymbol{x}_{0}\right)$. Además, $\tau_{0}<\tau\left(\boldsymbol{x}_{0}\right)$. Por lo tanto, para algunos $s$ en el intervalo $\left(\tau_{0}, \tau\left(\boldsymbol{x}_{0}\right)\right)$, se satisface que

$$
E_{\lambda}(\boldsymbol{x}(s))>E_{\lambda}\left(\boldsymbol{x}\left(\tau_{0}\right)\right)
$$

Sin embargo, $\frac{d E_{\lambda}(\boldsymbol{x}(t))}{d t}=\nabla E_{\lambda}(\boldsymbol{x}(t))^{T} \frac{d \boldsymbol{x}}{d t}=-\rho\left\|\nabla E_{\lambda}(\boldsymbol{x}(t))\right\|^{2} \leq 0$, lo cual significa que $\nabla E_{\lambda}$ es no creciente en $\left[\tau_{0}, \tau\left(\boldsymbol{x}_{0}\right)\right)$, lo que contradice (11). Por lo tanto, $\tau\left(\boldsymbol{x}_{0}\right)=+\infty$.

$\nabla$

El siguiente resultado establece la convergencia de la trayectoria solución.

Corolario 4.4. Sea $\boldsymbol{x}(t)$, con $t \in\left[t_{0} ; \tau\left(\boldsymbol{x}_{0}\right)\right)$, una solución maximal de $(7)$.

(i) Si $\tau\left(\boldsymbol{x}_{0}\right)=+\infty \quad y\{\boldsymbol{x}(t)\}$ es acotada, entonces

$$
\lim _{t \rightarrow+\infty} \nabla E_{\lambda}(\boldsymbol{x}(t))=0
$$

(ii) Si $F$ es una función $P_{0}$, entonces $\mathcal{L}\left(\boldsymbol{x}_{0}\right)$ es acotado y todo punto de acumulación de la trayectoria $\boldsymbol{x}(t)$ es solución del PCNL. 
Demostración. (i) Demostramos anteriormente que $\frac{d E_{\lambda}(\boldsymbol{x}(t))}{d t} \leq 0$, lo que implica que $E_{\lambda}(\boldsymbol{x}(t))$ es decreciente y es acotada inferiormente, ya que $E_{\lambda}(\boldsymbol{x}(t)) \geq 0$. Luego, existe un punto de acumulación $\boldsymbol{x}_{*}$, en el cual $E_{\lambda}$ alcanza un ínfimo; más aún, este punto es un minimizador de $E_{\lambda}$, lo cual implica que $\nabla E_{\lambda}\left(\boldsymbol{x}_{*}\right)=0$. Además, dado que $\tau\left(\boldsymbol{x}_{0}\right)=+\infty$, podemos pasar al límite; en efecto,

$$
0=\nabla E_{\lambda}\left(\boldsymbol{x}_{*}\right)=\nabla E_{\lambda}\left(\lim _{t \rightarrow+\infty} \boldsymbol{x}(t)\right)=\lim _{t \rightarrow+\infty} \nabla E_{\lambda}(\boldsymbol{x}(t)) .
$$

La hipótesis de que $\{\boldsymbol{x}(t)\}$ esté acotada garantiza que el sistema dinámico (7) tiene solución. En [18] se muestra un ejemplo en el que la conclusión del corolario no se tiene, si $\{\boldsymbol{x}(t)\}$ no es acotada.

(ii) Supongamos que $F$ es una función $P_{0}$; entonces $\mathcal{L}\left(\boldsymbol{x}_{0}\right)$ es acotado ([15]) y por tanto $\{\boldsymbol{x}(t)\}$ también es acotada. Luego si $\boldsymbol{x}_{*}$ es un punto de acumulación, $\nabla E_{\lambda}\left(\boldsymbol{x}_{*}\right)=0$, lo cual implica que $\boldsymbol{x}_{*}$ es un minimizador de $E_{\lambda}$ y que $F$ es una función $P_{0} ; \boldsymbol{x}_{*}$ también es solución del NCPL ([15]).

En lo que sigue, consideramos una solución $\boldsymbol{x}_{*}$ del PCNL, la cual por la Proposición 4.1 es también un punto de equilibrio de (7), y supondremos que $\boldsymbol{x}_{*}$ es un punto de equilibrio aislado de (7).

Teorema 4.5. Si $\boldsymbol{x}_{*}$ es un punto de equilibrio aislado de (7), $\boldsymbol{x}_{*}$ es estable asintóticamente para (7).

Demostración. Sean $\Omega_{*} \subseteq \mathbb{R}^{n}$ una vecindad abierta del punto de equilibrio aislado $\boldsymbol{x}_{*} \mathrm{y}$ $E_{\lambda}$ la función definida en (4), la cual es continuamente diferenciable y no negativa para todo $\boldsymbol{x} \in \mathbb{R}^{n}$. Debido a que $\boldsymbol{x}_{*}$ es una solución del PCNL,

$$
E_{\lambda}\left(\boldsymbol{x}_{*}\right)=0 .
$$

Supongamos que existe un $\boldsymbol{x} \in \Omega_{*}$ con $\boldsymbol{x} \neq \boldsymbol{x}_{*}$ tal que $E_{\lambda}(\boldsymbol{x})=0$. Por (6) tenemos que $\nabla E_{\lambda}(\boldsymbol{x})=V^{T} \Phi_{\lambda}(\boldsymbol{x})=0$, para toda $V \in \partial \Phi_{\lambda}(\boldsymbol{x})$, lo que implica que $\boldsymbol{x}$ es un punto de equilibrio de (7), contradiciendo la hipótesis de que $\boldsymbol{x}_{*}$ es un punto de equilibrio aislado de (7). Luego

$$
E_{\lambda}(\boldsymbol{x})>0, \quad \text { para todo } \boldsymbol{x} \in \Omega_{*}, \boldsymbol{x} \neq \boldsymbol{x}_{*} .
$$

Por otra parte tenemos que

$$
\frac{d E_{\lambda}(\boldsymbol{x}(t))}{d t}=\nabla_{\boldsymbol{x}(t)} E_{\lambda}(\boldsymbol{x}(t))^{T} \frac{d x}{d t}=-\left\|\nabla_{\boldsymbol{x}(t)} E_{\lambda}(\boldsymbol{x}(t))\right\|^{2} \leq 0 .
$$

Así, de (13), (14), (15) y de la Definición 3 tenemos que $E_{\lambda}$ es una función de Liapunov para (7) sobre $\Omega_{*}$.

Dado que $\boldsymbol{x}_{*}$ es un punto de equilibrio aislado del modelo (7), de (15) concluimos que

$$
\frac{d E_{\lambda}(\boldsymbol{x}(t))}{d t}<0, \text { para todo } \boldsymbol{x}(t) \in \Omega_{*}, \boldsymbol{x}(t) \neq \boldsymbol{x}_{*} .
$$

Por lo tanto, el Teorema 3.9 garantiza que $\boldsymbol{x}_{*}$ es estable asintóticamente para (7).

Vol. 34, No. 2, 2016] 
Diremos que una solución $\boldsymbol{x}_{*}$ de PCNL se llama regular, si todas las matrices del $\partial \Phi_{\lambda}\left(\boldsymbol{x}_{*}\right)$ son no singulares ([22]). Con frecuencia la regularidad conduce a convergencia superlineal de algunos métodos de optimización.

Un aspecto interesante del siguiente teorema es la relación del concepto de regularidad de una solución del PCNL con el de estabilidad exponencial. La demostración del teorema es análoga a la del Teorema 4.6 en [17] para la función de complementariedad de FischerBurmeister (que corresponde a $\varphi_{\lambda}$ con $\lambda=2$ ).

Teorema 4.6. Si $\boldsymbol{x}_{*}$ es una solución regular del PCNL, entonces $\boldsymbol{x}_{*}$ es estable exponencialmente para ( 7$)$.

Demostración. Dado que $\boldsymbol{x}_{*}$ es una solución regular del PCNL, existen ([23]) una vecindad $B\left(\boldsymbol{x}_{*} ; \delta\right)=\left\{\boldsymbol{x} \in \mathbb{R}^{n}:\left\|\boldsymbol{x}-\boldsymbol{x}_{*}\right\|<\delta\right\}$ de $\boldsymbol{x}_{*}$ y una constante $C$ tal es que, para todo $\boldsymbol{x}$ en dicha vecindad y cualquier $V \in \partial \Phi_{\lambda}(\boldsymbol{x})$, la matriz $V$ es no singular y

$$
\text { máx }\left\{\|V\|,\left\|V^{-1}\right\|\right\} \leq C \text {. }
$$

Sea $\delta>0$ suficientemente pequeño tal que, para cualquier $\boldsymbol{x}\left(t_{0}\right) \in \mathcal{B}\left(\boldsymbol{x}_{*} ; \delta\right)$, se tiene que $\boldsymbol{x}(t) \rightarrow \boldsymbol{x}_{*}$ siempre que $t \rightarrow \infty$, y de modo que el resultado del párrafo anterior se satisfaga. Por tanto, existen $\kappa_{1}$ y $\kappa_{2}$ tales que para todo $\boldsymbol{x} \in \mathcal{B}\left(\boldsymbol{x}_{*} ; \delta\right)$,

$$
\kappa_{1}\|\boldsymbol{v}\|^{2} \leq \boldsymbol{v}^{T} V^{T} V \boldsymbol{v} \leq \kappa_{2}\|\boldsymbol{v}\|^{2} .
$$

Por otra parte, si $\boldsymbol{x}_{*}$ es una solución regular del PCNL, entonces es una solución aislada de $\Phi_{\lambda}(\boldsymbol{x})=0$ ([22], [23]). Luego, de (9), $\boldsymbol{x}_{*}$ es un punto de equilibrio aislado de (7), lo cual por el Teorema 4.5 implica que $\boldsymbol{x}_{*}$ es estable asintóticamente.

Dado que la función $\Phi_{\lambda}$ es semisuave ([15]), se tiene que para todo $V \in \partial \Phi_{\lambda}(\boldsymbol{x})$

$$
\Phi_{\lambda}(\boldsymbol{x})=\Phi_{\lambda}\left(\boldsymbol{x}_{*}\right)+V\left(\boldsymbol{x}-\boldsymbol{x}_{*}\right)+o\left(\left\|\boldsymbol{x}-\boldsymbol{x}_{*}\right\|\right) .
$$

Observemos que, $o\left(\left\|\boldsymbol{x}-\boldsymbol{x}_{*}\right\|\right)=H(\boldsymbol{x})$, donde $H$ es un campo vectorial sobre $\mathbb{R}^{n}$. Esto significa que

$$
\lim _{\boldsymbol{x} \rightarrow \boldsymbol{x}_{*}} \frac{\|H(\boldsymbol{x})\|}{\left\|\boldsymbol{x}-\boldsymbol{x}_{*}\right\|}=0
$$

lo cual implica que, para todo $\epsilon>0$, existe $\bar{\delta}>0$ tal que

$$
\frac{\|H(\boldsymbol{x})\|}{\left\|\boldsymbol{x}-\boldsymbol{x}_{*}\right\|} \leq \epsilon, \quad \text { para todo } \boldsymbol{x} \in B\left(\boldsymbol{x}_{*} ; \bar{\delta}\right)
$$

Equivalentemente,

$$
o\left(\left\|\boldsymbol{x}-\boldsymbol{x}_{*}\right\|\right) \leq \epsilon\left\|\boldsymbol{x}-\boldsymbol{x}_{*}\right\| \quad \text { para todo } \boldsymbol{x} \in B\left(\boldsymbol{x}_{*} ; \bar{\delta}\right) .
$$

En particular, la condición (19) se satisface para algún $0<\epsilon<\kappa_{1}$ y para todo $x$ en $B\left(\boldsymbol{x}_{*} ; \delta\right)$. Aquí hemos usado el hecho de que (ver Figura 3 ), si $\bar{\delta} \geq \delta$, entonces la condición (19) se satisface para todo $\boldsymbol{x} \in B\left(\boldsymbol{x}_{*} ; \delta\right)$; y si $\bar{\delta}<\delta$, entonces reducimos el tamaño de $\delta$ para que (19) se satisfaga. 

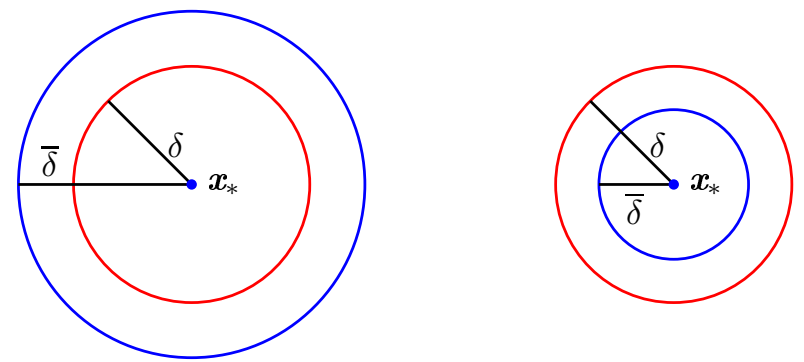

Figura 3. Reduciendo el tamaño de $\delta$ si fuese necesario.

Ahora consideremos la función $\Gamma$ definida por $\Gamma(t)=\left\|\boldsymbol{x}(t)-\boldsymbol{x}_{*}\right\|^{2}$, para todo $t \in$ $\left[t_{0}, \infty\right)$. Derivando $\Gamma$ respecto a $t$ y usando (6), tenemos que, para todo $V \in \partial \Phi_{\lambda}(\boldsymbol{x}(t))$,

$$
\frac{d \Gamma(t)}{d t}=2\left[\boldsymbol{x}(t)-\boldsymbol{x}_{*}\right]^{T} \frac{d \boldsymbol{x}}{d t}=-2\left[\boldsymbol{x}(t)-\boldsymbol{x}_{*}\right]^{T} V^{T} \Phi_{\lambda}(\boldsymbol{x}(t)) .
$$

Supongamos que $T=\left\{t \in\left[t_{0}, \infty\right) \mid:\left\|\boldsymbol{x}(t)-\boldsymbol{x}_{*}\right\| \geq \delta\right\} \neq \emptyset$. El conjunto $T$ es acotado inferiormente, luego existe $\bar{\tau}=\inf T$, que corresponde al primer valor de $t$ para el cual $\boldsymbol{x}(t) \notin B\left(\boldsymbol{x}_{*} ; \delta\right)$.

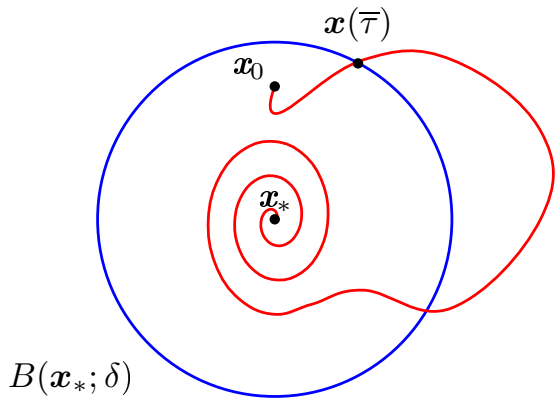

Figura 4. $\boldsymbol{x}_{*}$ es asintóticamente estable, pero no exponencialmente estable.

Sustituyendo (18) en (21) y teniendo en cuenta que $\Phi_{\lambda}\left(\boldsymbol{x}_{*}\right)=0$, tenemos que para todo $t \in \bar{I}=\left[t_{0}, \bar{\tau}\right)$,

$$
\frac{d \Gamma(t)}{d t}=-2\left(\boldsymbol{x}(t)-\boldsymbol{x}_{*}\right)^{T} V^{T} V\left(\boldsymbol{x}(t)-\boldsymbol{x}_{*}\right)+(-2)\left(\boldsymbol{x}(t)-\boldsymbol{x}_{*}\right)^{T} V^{T} o\left(\left\|\boldsymbol{x}(t)-\boldsymbol{x}_{*}\right\|\right) .
$$

Usando (17) se tiene que

$$
-2\left(\boldsymbol{x}(t)-\boldsymbol{x}_{*}\right)^{T} V^{T} V\left(\boldsymbol{x}(t)-\boldsymbol{x}_{*}\right) \leq-2 \kappa_{1}\left\|\boldsymbol{x}(t)-\boldsymbol{x}_{*}\right\|^{2} .
$$

El segundo sumando de (21) satisface la desigualdad

$$
(-2)\left(\boldsymbol{x}(t)-\boldsymbol{x}_{*}\right)^{T} V^{T} o\left(\left\|\boldsymbol{x}(t)-\boldsymbol{x}_{*}\right\|\right) \leq \epsilon\left\|\boldsymbol{x}(t)-\boldsymbol{x}_{*}\right\|^{2},
$$

porque $g(\boldsymbol{x})=(-2)\left(\boldsymbol{x}(t)-\boldsymbol{x}_{*}\right)^{T} V^{T} o\left(\left\|\boldsymbol{x}(t)-\boldsymbol{x}_{*}\right\|\right)=o\left(\left\|\boldsymbol{x}(t)-\boldsymbol{x}_{*}\right\|^{2}\right)$, ya que

$$
\lim _{\boldsymbol{x}(t) \rightarrow \boldsymbol{x}_{*}} \frac{|g(\boldsymbol{x})|}{\left\|\boldsymbol{x}(t)-\boldsymbol{x}_{*}\right\|^{2}}=0 .
$$

Vol. 34, No. 2, 2016] 
En efecto,

$$
\begin{aligned}
\lim _{\boldsymbol{x}(t) \rightarrow \boldsymbol{x}_{*}} \frac{|g(\boldsymbol{x})|}{\left\|\boldsymbol{x}(t)-\boldsymbol{x}_{*}\right\|^{2}} & \leq \lim _{\boldsymbol{x}(t) \rightarrow \boldsymbol{x}_{*}} \frac{2\|V\|\left\|\left(\boldsymbol{x}(t)-\boldsymbol{x}_{*}\right)\right\| o\left(\left\|\boldsymbol{x}(t)-\boldsymbol{x}_{*}\right\|\right)}{\left\|\boldsymbol{x}(t)-\boldsymbol{x}_{*}\right\|\left\|\boldsymbol{x}(t)-\boldsymbol{x}_{*}\right\|} \\
& \leq 2\|V\| \lim _{\boldsymbol{x}(t) \rightarrow \boldsymbol{x}_{*}} \frac{o\left(\left\|\boldsymbol{x}(t)-\boldsymbol{x}_{*}\right\|\right)}{\left\|\boldsymbol{x}(t)-\boldsymbol{x}_{*}\right\|} \leq 2(C)(0)=0
\end{aligned}
$$

donde $C$ es la constante dada en (16).

Luego, por (21), (22) y (23) tenemos que

$$
\frac{d \Gamma(t)}{d t} \leq\left(-2 \kappa_{1}+\epsilon\right)\left\|\boldsymbol{x}(t)-\boldsymbol{x}_{*}\right\|^{2}=\left(-2 \kappa_{1}+\epsilon\right) \Gamma(t) .
$$

Usando el Corolario 2.1 en [27] tenemos que $\Gamma(t) \leq e^{\left(-2 \kappa_{1}+\epsilon\right) t} \Gamma\left(t_{0}\right)$, para todo $t \in \bar{I}$, o equivalentemente,

$$
\left\|\boldsymbol{x}(t)-\boldsymbol{x}_{*}\right\| \leq e^{\omega t}\left\|\boldsymbol{x}\left(t_{0}\right)-\boldsymbol{x}_{*}\right\|, \quad t \in \bar{I},
$$

con $\omega=-\kappa_{1}+\epsilon / 2<0$. Tomando el límite superior en (24), tenemos que

$$
\delta \leq \limsup _{t \uparrow \bar{\tau}}\left\|\boldsymbol{x}(t)-\boldsymbol{x}_{*}\right\| \leq e^{\omega \bar{\tau}}\left\|\boldsymbol{x}\left(t_{0}\right)-\boldsymbol{x}_{*}\right\|<\delta,
$$

lo cual es una contradicción. Luego, $T=\emptyset$ e $\bar{I}=[0,+\infty)$, y por la desigualdad (24) se completa la prueba de la estabilidad exponencial de $\boldsymbol{x}_{*}$.

Del teorema anterior tenemos que para todo $t \in[0,+\infty)$ se satisface la desigualdad (24). En particular, para $t=k \in \mathbb{N}$ con $\boldsymbol{x}(k):=\boldsymbol{x}_{k}$ :

$$
\left\|\boldsymbol{x}_{k}-\boldsymbol{x}_{*}\right\| \leq e^{\omega k}\left\|\boldsymbol{x}_{0}-\boldsymbol{x}_{*}\right\|
$$

esto garantiza la convergencia de cualquier sucesión que aproxime la trayectoria solución del modelo (7), como se ilustra en la simulación numérica que presentamos en la siguiente sección.

\section{Pruebas numéricas}

En esta sección analizamos numéricamente el algoritmo propuesto. Para ello usamos siete problemas de complementariedad asociados a algunas funciones de prueba ampliamente utilizadas en la literatura sobre complementariedad, las cuales definimos a continuación (entre paréntesis aparece la abreviatura que utilizaremos en las tablas de resultados para hacer referencia a cada problema), con su solución $\left(\boldsymbol{x}_{*}\right)$ y el punto inicial usado en los algoritmos $\left(\boldsymbol{x}_{0}\right)$.

Problema de Billups (Billups): $F: \mathbb{R} \rightarrow \mathbb{R}$ está definida como

$$
F(\boldsymbol{x})=(x-1)^{2}-1.1
$$

$$
\boldsymbol{x}^{*}=2.0488 \text { y } \boldsymbol{x}_{0}=0 .
$$


Problema de Kojima-Shindo (Koj-Shi): $F: \mathbb{R}^{4} \rightarrow \mathbb{R}^{4}$ está definida como

$$
\begin{gathered}
F(\boldsymbol{x})=\left(\begin{array}{c}
3 x_{1}^{2}+2 x_{1} x_{2}+2 x_{2}^{2}+x_{3}+3 x_{4}-6 \\
2 x_{1}^{2}+x_{2}^{2}+x_{1}+3 x_{3}+2 x_{4}-2 \\
3 x_{1}^{2}+x_{1} x_{2}+2 x_{2}^{2}+2 x_{3}+3 x_{4}-1 \\
x_{1}^{2}+3 x_{2}^{2}+2 x_{3}+3 x_{4}-3
\end{array}\right) ; \\
\boldsymbol{x}^{*}=\left(\begin{array}{llll}
\sqrt{6} / 2 & 0 & 0 & 0.5
\end{array}\right)^{T} \text { y } \boldsymbol{x}_{0}=\left(\begin{array}{llll}
0 & 0 & 0 & 0
\end{array}\right)^{T} .
\end{gathered}
$$

Problema de Kojima-Shindo modificado (Koj-Shi-mod): $F: \mathbb{R}^{4} \rightarrow \mathbb{R}^{4}$,

$$
\begin{gathered}
F(\boldsymbol{x})=\left(\begin{array}{c}
3 x_{1}^{2}+2 x_{1} x_{2}+2 x_{2}^{2}+x_{3}+3 x_{4}-6 \\
2 x_{1}^{2}+x_{2}^{2}+x_{1}+10 x_{3}+2 x_{4}-2 \\
3 x_{1}^{2}+x_{1} x_{2}+2 x_{2}^{2}+2 x_{3}+9 x_{4}-9 \\
x_{1}^{2}+3 x_{2}^{2}+2 x_{3}+3 x_{4}-3
\end{array}\right) ; \\
\boldsymbol{x}^{*}=\left(\begin{array}{llll}
\sqrt{6} / 2 & 0 & 0 & 0.5
\end{array}\right)^{T} \text { у } \boldsymbol{x}_{0}=\left(\begin{array}{llll}
0 & 0 & 0 & 0
\end{array}\right)^{T} .
\end{gathered}
$$

Problema de Kojima-Josephy (Koj-Jo): $F: \mathbb{R}^{4} \rightarrow \mathbb{R}^{4}$, definida como

$$
\begin{aligned}
& F(\boldsymbol{x})=\left(\begin{array}{c}
3 x_{1}^{2}+2 x_{1} x_{2}+2 x_{2}^{2}+x_{3}+3 x_{4}-6 \\
2 x_{1}^{2}+x_{2}^{2}+x_{1}+3 x_{3}+2 x_{4}-2 \\
3 x_{1}^{2}+x_{1} x_{2}+2 x_{2}^{2}+2 x_{3}+3 x_{4}-9 \\
x_{1}^{2}+3 x_{2}^{2}+2 x_{3}+3 x_{4}-3
\end{array}\right) ; \\
& \boldsymbol{x}^{*}=\left(\begin{array}{llll}
1 & 0 & 3 & 0
\end{array}\right)^{T} \text { y } \boldsymbol{x}_{0}=\left(\begin{array}{llll}
0 & 0 & 0 & 0
\end{array}\right)^{T} .
\end{aligned}
$$

Problema de Mathiesen Modificado (Mathiesen): $F: \mathbb{R}^{4} \rightarrow \mathbb{R}^{4}$,

$$
F(\boldsymbol{x})=\left(\begin{array}{c}
-x_{2}+x_{3}+x_{4} \\
x_{1}-\frac{4.5 x_{3}+2.7 x_{4}}{x_{2}+1} \\
5-x_{1}-\frac{0.5 x_{3}+0.3 x_{4}}{x_{3}+1} \\
3-x_{1}
\end{array}\right)
$$

$$
\boldsymbol{x}^{*}=\left(\begin{array}{llll}
a & 0 & 0 & 0
\end{array}\right)^{T}, a \in[0,3] \text { y } \boldsymbol{x}_{0}=\left(\begin{array}{llll}
1 & 1 & 1 & 1
\end{array}\right)^{T} .
$$

Problema tomado de [17] (cuadrático): $F: \mathbb{R}^{2} \rightarrow \mathbb{R}^{2}$ definida como

$$
\begin{array}{r}
F(\boldsymbol{x})=\left(\begin{array}{l}
2 x_{1}+4 x_{2} \\
2 x_{2}+4 x_{1}
\end{array}\right) ; \\
\boldsymbol{x}^{*}=\left(\begin{array}{ll}
0 & 0
\end{array}\right)^{T} \text { у } \boldsymbol{x}_{0}=\left(\begin{array}{ll}
1 & 1
\end{array}\right)^{T} .
\end{array}
$$

A continuación presentamos dos algoritmos que componen el modelo de red neuronal propuesto para resolver el PCNL. El primero describe un procedimiento para calcular una matriz $V \in \partial \Phi_{\lambda}(\boldsymbol{x})$ análogo al usado en [9] para la función de Fischer, y cuya idea general consiste en considerar la sucesión de vectores $\left\{\boldsymbol{y}_{k}=\boldsymbol{x}+\varepsilon_{k} \boldsymbol{z}\right\}$, donde $\left\{\varepsilon_{k}\right\}$ es una sucesión de números positivos que converge a cero y $\boldsymbol{z}$ es un vector tal que $z_{i}=1$

Vol. 34, No. 2, 2016] 
si $F_{i}(\boldsymbol{x})=x_{i}=0$, y $z_{i}=0$ en otro caso. La sucesión así construida converge a $\boldsymbol{x}$ y las matrices jacobianas $\Phi_{\lambda}^{\prime}\left(\boldsymbol{y}_{k}\right)$ existen. Esta deducción se encuentra detalladamente en [1] y [15].

El segundo algoritmo constituye propiamente el modelo de red neuronal propuesto para resolver el PCNL. Dentro de este algoritmo implementamos una versión matricial del método de Runge-Kutta de cuarto orden ([5]), cuya iteración básica es de la forma

$$
\boldsymbol{x}_{k+1}=\boldsymbol{x}_{k}+\frac{h}{6} M\left[\begin{array}{llll}
1 & 2 & 2 & 1
\end{array}\right]^{T},
$$

donde $M$ es una matriz de tamaño $n \times 4$, siendo $n$ la dimensión del problema.
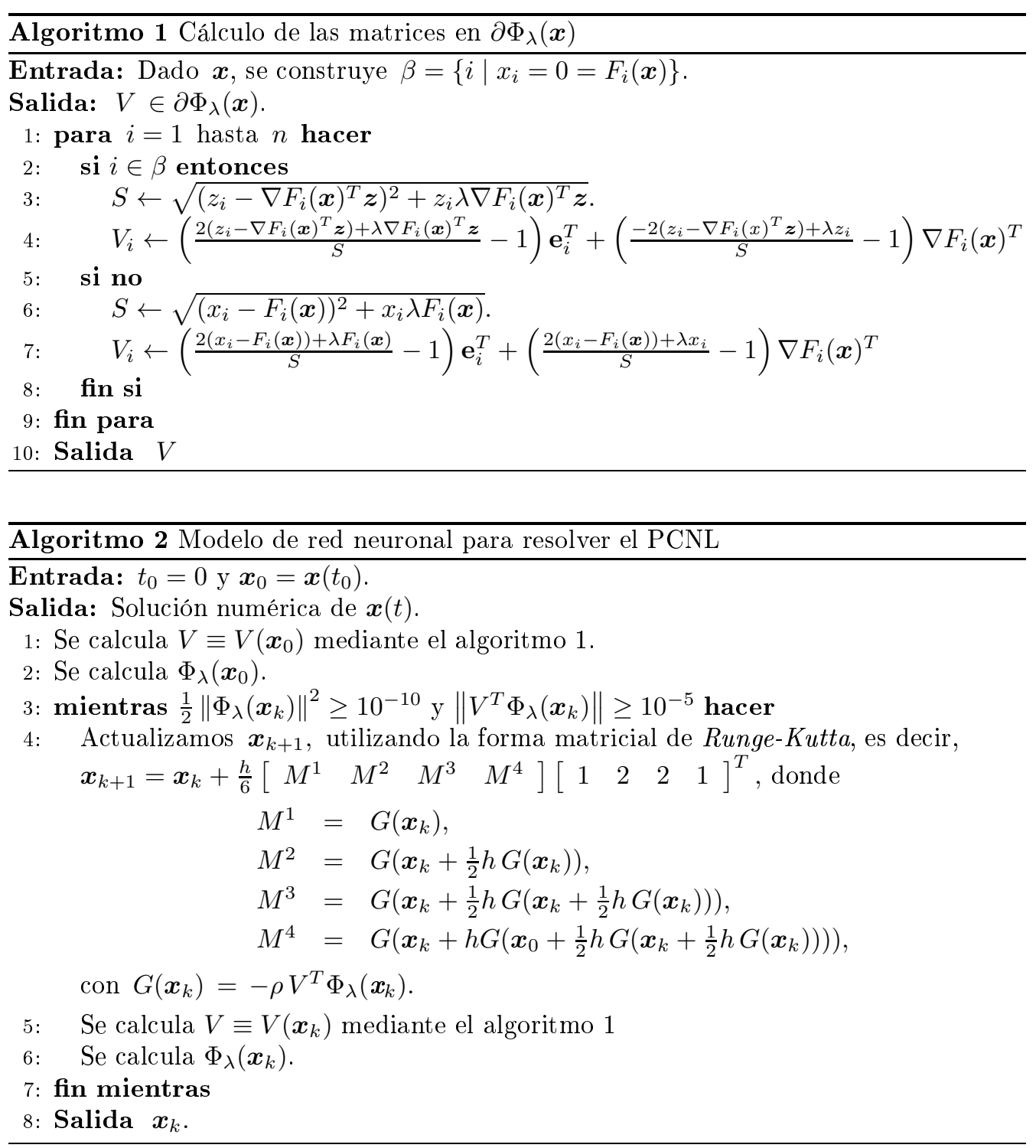
Para escribir los códigos de los algoritmos utilizamos el software MATLAB ${ }^{\circledR}$. Realizamos los experimentos numéricos en un computador con procesador Intel(R) Core(TM) i5$3450 \mathrm{~S}$ CPU de $2,80 \mathrm{GHz}$ en un sistema operativo de 64 bits.

La Tabla 1 contiene los resultados obtenidos con el Algoritmo 2, el cual usa un parámetro $\lambda$ dinámico ([15]) y los obtenidos con un algoritmo que usa un modelo análogo al propuesto en [17], en el cual $\lambda=2$. Estos últimos resultados se indican en la tabla usando un asterisco después de la abreviatura del problema. Para todos los experimentos, el valor del parámetro $\rho$, en el modelo, es $\rho=10^{3}$, y el tamaño de paso escogido en el método de Runge-Kutta es $h=10^{-5}$.

La primera columna de la tabla indica el problema utilizado; la segunda, da el valor del parámetro $t$ en el cual declaramos convergencia, y las tres columnas siguientes contienen los valores finales de $\left\|\boldsymbol{x}(t)-\boldsymbol{x}^{*}\right\|,\left\|\nabla E_{\lambda}(\boldsymbol{x}(t))\right\|$ y $\left|E_{\lambda}(\boldsymbol{x}(t))\right|$, respectivamente.

\begin{tabular}{|l|c|c|c|c|}
\hline \hline Problema & $t$ & $\left\|x(t)-x^{*}\right\|$ & $\left\|\nabla E_{\lambda}\right\|$ & $\left|E_{\lambda}\right|$ \\
\hline \hline Billups & $1,270000 \mathrm{e}-03$ & $2,398465 \mathrm{e}-06$ & $5,675707 \mathrm{e}-05$ & $9,151717 \mathrm{e}-11$ \\
\hline Billups* & $3,010000 \mathrm{e}-03$ & $2,161289 \mathrm{e}-06$ & $2,942230 \mathrm{e}-05$ & $9,837168 \mathrm{e}-11$ \\
\hline Koj-Shi & $4,931000 \mathrm{e}-03$ & $2,113732 \mathrm{e}-05$ & $1,888083 \mathrm{e}-05$ & $9,977221 \mathrm{e}-11$ \\
\hline Koj-Shi* & $2,199000 \mathrm{e}-02$ & $2,229580 \mathrm{e}-05$ & $9,957821 \mathrm{e}-06$ & $1,110084 \mathrm{e}-10$ \\
\hline Koj-Shi-mod & $5,249000 \mathrm{e}-03$ & $2,010010 \mathrm{e}-05$ & $1,892561 \mathrm{e}-05$ & $9,977325 \mathrm{e}-11$ \\
\hline Koj-Shi-mod $^{*}$ & $2,327000 \mathrm{e}-02$ & $2,061545 \mathrm{e}-05$ & $9,987753 \mathrm{e}-06$ & $1,029506 \mathrm{e}-10$ \\
\hline Koj-Jos $^{*}$ & $9,847000 \mathrm{e}-03$ & $2,761663 \mathrm{e}-05$ & $1,447005 \mathrm{e}-05$ & $9,990373 \mathrm{e}-11$ \\
\hline Koj-Jos* $^{*}$ & $4,337000 \mathrm{e}-02$ & $3,812819 \mathrm{e}-05$ & $9,988814 \mathrm{e}-06$ & $1,904285 \mathrm{e}-10$ \\
\hline Mathiesen & $6,669000 \mathrm{e}-02$ & $3,005689 \mathrm{e}-02$ & $9,999414 \mathrm{e}-06$ & $3,586985 \mathrm{e}-10$ \\
\hline Mathiesen* & $2,600000 \mathrm{e}-02$ & $2,990021 \mathrm{e}-02$ & $9,976923 \mathrm{e}-06$ & $1,569724 \mathrm{e}-09$ \\
\hline Cuadrático $^{*}$ & $2,860000 \mathrm{e}-03$ & $1,525697 \mathrm{e}-05$ & $2,798854 \mathrm{e}-05$ & $9,791978 \mathrm{e}-11$ \\
\hline Cuadrático $^{*}$ & $1,359000 \mathrm{e}-02$ & $1,530965 \mathrm{e}-05$ & $1,288038 \mathrm{e}-05$ & $9,859705 \mathrm{e}-11$ \\
\hline
\end{tabular}

Cuadro 1. Resultados con $\lambda$ dinámico y con $\lambda=2$.

Esta tabla muestra que el modelo propuesto es competitivo, y en el caso del problema cuadrático es mejor. Cabe mencionar que el problema de Billups fue construido por Billups ([4]) con el objetivo de hacer que los métodos usados para resolverlo fallen ([15]); es decir, es un problema de alta exigencia en cuanto a su convergencia, pero observamos que los algoritmos lo resolvieron.

\section{Conclusiones}

En este artículo proponemos un modelo de red neuronal basado en el método de máximo descenso para resolver problemas de complementariedad no lineal, el cual usa por primera vez una familia uniparamétrica de funciones de complementariedad como función energía. Establecemos resultados de existencia, estabiliad y de convergencia de la trayectoria

Vol. 34, No. 2, 2016] 
de la red neuronal. Además, presentamos algunas pruebas numéricas preliminares que muestran que el modelo propuesto es competitivo.

Finalmente, en búsqueda de ampliar el espectro de trabajo con respecto a redes neuronales para complementariedad no lineal, consideramos conveniente realizar más pruebas numéricas del algoritmo propuesto y modificar el modelo propuesto para resolver problemas con restricciones.

\section{Agradecimientos}

Los autores agradecen a la Universidad del Cauca por el tiempo concedido para esta investigación mediante el Proyecto de investigación VRI ID 4189, y a un árbitro anónimo por sus sugerencias y comentarios que ayudaron a mejorar la presentación y contenido de este artículo.

\section{Referencias}

[1] Arenas F., Martínez H.J. and Pérez R., "Least change secant update methods for nonlinear complementarity problem", Ingeniería y Ciencia 11 (2015), 11-36.

[2] Arenas F., Martínez H.J. \& Pérez R., "Redefinición de la función de complementariedad de Kanzow", Revista de Ciencias 18 (2014), No. 2, 111-122.

[3] Arias C.A., Un algoritmo cuasi Newton global para problemas de complementariedad no lineal, Tesis de Maestría, Universidad del Cauca, 2014, 44 p.

[4] Billups S. C., Algorithms for complementarity problems and generalized equations, Thesis (Ph.D.), University of Wisconsin, 1995.

[5] Burden R.L. \& Douglas Faires J., Análisis Numérico, 7ma ed., Thomson Learning, 2002.

[6] Chen J-S., Ko C-H. and Pan S., "A Neural network based on the generalized FisherBurmeister function for nonlinear complementarity problems", Information Sciences 180 (2010), No. 5, 697-711.

[7] Chen J-S. and Pan S., "A family of NCP functions and a descent method for the nonlinear complementarity problem", Comput. Optimi. Appl. 40 (2008), No. 3, 389-404.

[8] Clarke F.H., Optimization and nonsmooth analysis, John Wiley \& Sons, Inc., New York, 1983.

[9] De Luca T., Facchinei F. and Kanzow C., "A semismooth equation approach to the solution of nonlinear complementarity problems", Math. Programming 75 (1996), No. 3, Ser. A, 407439.

[10] Dennis J.E., Jr. and Schnabel R.B., Numerical methods for unconstrained optimization and nonlinear equations, Prentice Hall, Inc., Englewood Cliffs, NJ, 1983.

[11] Ding J. and Yin H., "A new homotopy method for solving non-linear complementarity problems", Numer. Math. J. Chi. Univ. (Engl. Ser.) 16 (2007), No. 2, 155-163.

[12] Ferris M.C. and Pang J.S., "Engineering and economic applications of complementarity problems", SIAM Rev. 39 (1997), No. 4, 669-713.

[Revista Integración 
[13] Fischer A., "A special Newton-type optimization method", Optimization 24 (1992), No. 3-4, 269-284.

[14] Hopfield J. and Tank D.W., "Neural computation of decisions in optimization problems", Biol. Cybernet. 52 (1985), No. 3, 141-152.

[15] Kanzow C.J. and Kleinmichel H., "A new class of semismooth Newton-type methods for nonlinear complementarity problems", Computat. Optimi. Appl. 11 (1998), No. 3, 227-251.

[16] Kostreva M., "Elasto-hdrodynamic lubrication: An non-linear complementarity problem", Internat. J. Numer. Methods Fluids 4 (1984), No. 4, 377-397.

[17] Liao L-Z, Qi H. and Qi L., "Solving nonlinear complementarity problems with neural networks: a reformulation method approach", J. Comput. Appl. Math. 131 (2001), No. 1-2, 343-359.

[18] Lillo W.E., Loh M.H., Hui S. and Zak S.H., "On solving constrained optimization problems with neural networks: apenalty method approach", IEEE Trans.Neural Networks 4 (1993), No. 6, 931-940.

[19] Nocedal J. and Wright S.J., Numerical optimization, Second ed., Springer, New York, 2006.

[20] Pang J-S. and Qi L.Q., "Nonsmooth equations: motivation and algorithms", SIAM J. Optim. 3 (1993), No. 3, 443-465.

[21] Pérez R., Lopes V.L.R. and Martínez J.M., "On the local convergence of quasi-Newton methods for solving non linear complementarity problems", Appl. Numer. Math. 30 (1999), No. 1, 3-22.

[22] Qi L.Q., "Convergence analysis of some algorithms for solving nonsmooth equations", Math. Oper. Research 18 (1993), No. 1, 227-244.

[23] Qi L.Q. and Sun J., "A nonsmooth version of Newton method", Math. Programming, 58 (1993), No. 3, Ser. A, 353-368.

[24] Wardrop J.G., "Some theorical aspects of road traffic research", Proceedings of the ICE 1 (1952), No. 3, 325-362.

[25] Watkins D.S., Fundamentals of matrix computations, Second ed., Wiley-Interscience, New York, 2002.

[26] Xu Q. and Dang C., "A new homotopy method for solving non-linear complementarity problems", Optimization 57 (2008), No. 5, 681-689.

[27] Zabczyk J., Mathematical control theory: an introduction, Birkhäuser, Boston Inc., Boston, MA, 1992.

Vol. 34, No. 2, 2016] 